\title{
Geodinámica y ocupación humana del litoral pacífico en el sur de Colombia y en el Ecuador desde el Holoceno (últimos 10000 años)
}

Géodynamique et occupation humaine des littoraux pacifiques équatorien et du Sud colombien depuis l'Holocène (derniers 10000 ans)

Holocene geodynamics and the human occupation on the Pacific coast of South Colombia and Ecuador over the last 10000 years

\section{Pierre Usselmann}

\section{OpenEdition}

Journals

Edición electrónica

URL: http://journals.openedition.org/bifea/1798

DOI: $10.4000 /$ bifea. 1798

ISSN: 2076-5827

Editor

Institut Français d'Études Andines

Edición impresa

Fecha de publicación: 1 diciembre 2010

Paginación: 589-602

ISSN: 0303-7495

Referencia electrónica

Pierre Usselmann, « Geodinámica y ocupación humana del litoral pacífico en el sur de Colombia y en el Ecuador desde el Holoceno (últimos 10000 años) », Bulletin de l'Institut français d'études andines [En línea], 39 (3) | 2010, Publicado el 01 junio 2011, consultado el 11 diciembre 2020. URL : http:// journals.openedition.org/bifea/1798; DOI : https://doi.org/10.4000/bifea.1798

\section{(c) (1)}

Les contenus du Bulletin de l'Institut français d'études andines sont mis à disposition selon les termes de la licence Creative Commons Attribution - Pas d'Utilisation Commerciale - Pas de Modification 4.0 International. 


\title{
Geodinámica y ocupación humana del litoral pacífico en el sur de Colombia y en el Ecuador desde el Holoceno (últimos 10000 años)
}

\author{
Pierre Usselmann*
}

\section{Resumen}

Las costas pacíficas del sur de Colombia y del Ecuador son ricas en restos de ocupación humana a veces todavía mal conocidos. Se trata a menudo de litorales bastante inhóspitos (caso de Colombia) y siempre de regiones, que han presentado, hoy como ayer, las huellas de importantes catástrofes naturales. Se puede enumerar rápidamente la presencia de capas de cenizas (riesgo volcánico), las consecuencias de movimientos sísmicos y, ligados, de tsunamis, los movimientos en masa en las laderas y las consecuencias del ahora bastante bien conocido fenómeno del Niño (inundaciones), y del elemento contrario, la Niña (gran sequía). Se puede pensar que las poblaciones costeras han tenido que adaptarse a tales amenazas, pero aquellas han podido llegar a veces a un punto en el que los establecimientos y los cultivos han sido totalmente destruidos, llegando al colapso de la presencia humana. Se propone ilustrar esto, desde un punto de vista geomorfológico, a partir de unos ejemplos que brinda la arqueología.

Palabras clave: Colombia, el Ecuador, costa pacífica, geodínamica, Holoceno

\section{Géodynamique et occupation humaine des littoraux} pacifiques équatorien et du Sud colombien depuis l'Holocène (derniers 10000 ans)

\section{Résumé}

Les côtes pacifiques du sud de la Colombie et de l'Équateur sont riches de restes d'occupations humaines souvent mal connus. Il s'agit parfois de littoraux inhospitaliers comme en Colombie,

* Director emerito de investigación, Centro Nacional de Investigaciones Cientificas (CNRS), UMR ESPACE, 24 Ter Rue Lakanal, 34090 Montpellier, Francia. E-Mail: pierre.usselmann@wanadoo.fi 
mais toujours d'environnements, dans le passé et encore actuellement, très marqués par les traces d'importants aléas naturels. On peut citer immédiatement la présence de couches de cendres (risque volcanique), les conséquences de mouvements sismiques et, liés, de tsunamis, les mouvements de masse sur les versants et, enfin, les conséquences du phénomène ENSO, maintenant bien connu par ses inondations (el Niño) et la sécheresse caractéristique de son contraire (la Niña). On peut penser que si les populations côtières ont dû s'adapter à ces contraintes, elles ont été parfois confrontées à des situations de destruction totale des cultures et des habitations aboutissant à la disparition de peuplements. Nous nous proposons d'illustrer ces propos d'un point de vue géomorphologique à partir de quelques exmples observés en archéologie.

Mots clés : Colombie, Équateur, côte pacifique, géodynamique, Holocène

\section{Holocene geodynamics and the human occupation on the Pacific} coast of South Colombia and Ecuador over the last 10000 years

\section{Abstract}

The Pacific coasts of South Colombia and Ecuador hold the remains of many human occupation, some of which are still unknown. The coast is sometimes inhospitable as in the Colombian case and often strongly affected by important natural hazards. Among these we can rapidly cite the risk from volcanic ash, the consequences of tectonics movements, tsunamis, mass movements of debris on slopes and consequences of well known Niño phenomena (hoods), and its counterpart the Niña (drought). The are totally destroyed resulting in collapse. Our purpose here is to llustrate from an geomorphogic

Key words: Colombia, Ecuador, Pacific coast, geodynamics, Holocene

Esta porción del litoral pacífico ecuatorial (fig. 1) se caracteriza a menudo por una inhospitalidad aparente. Esta se traduce por la presencia de una tupida y cerrada vegetación de manglares en el norte, y por la aridez de la costa hacia el sur. En contraste, se contempla el contacto con un océano rico de producción halieutica, lo que ha motivado la pronta instalación, y en gran parte bastante continua en el tiempo, de varias poblaciones cuyos restos constituyen ahora el objeto de investigaciones arqueológicas (Bouchard, 2008). Sea este litoral rocoso, con acantilados, o muy llano, con playas cubiertas de manglares o abiertas con lagunas, siempre existen importantes amenazas naturales que podemos clasificar rápidamente en tres tipos, los cuales originan a su vez peculiares procesos evolutivos:

- los movimientos tectónicos caracterizan un conjunto afectado en su totalidad por grandes fracturas, traduciéndose por la existencia de sismos y sus consecuencias;

- ligada a la tectónica, la omnipresencia del volcanismo afecta a toda la región, y, tratándose de la costa, particularmente las lluvias de cenizas;

- este litoral ha sido también afectado por los cambios climáticos planetarios, lo que provocó, muy en particular, variaciones del nivel del océano. Se debe

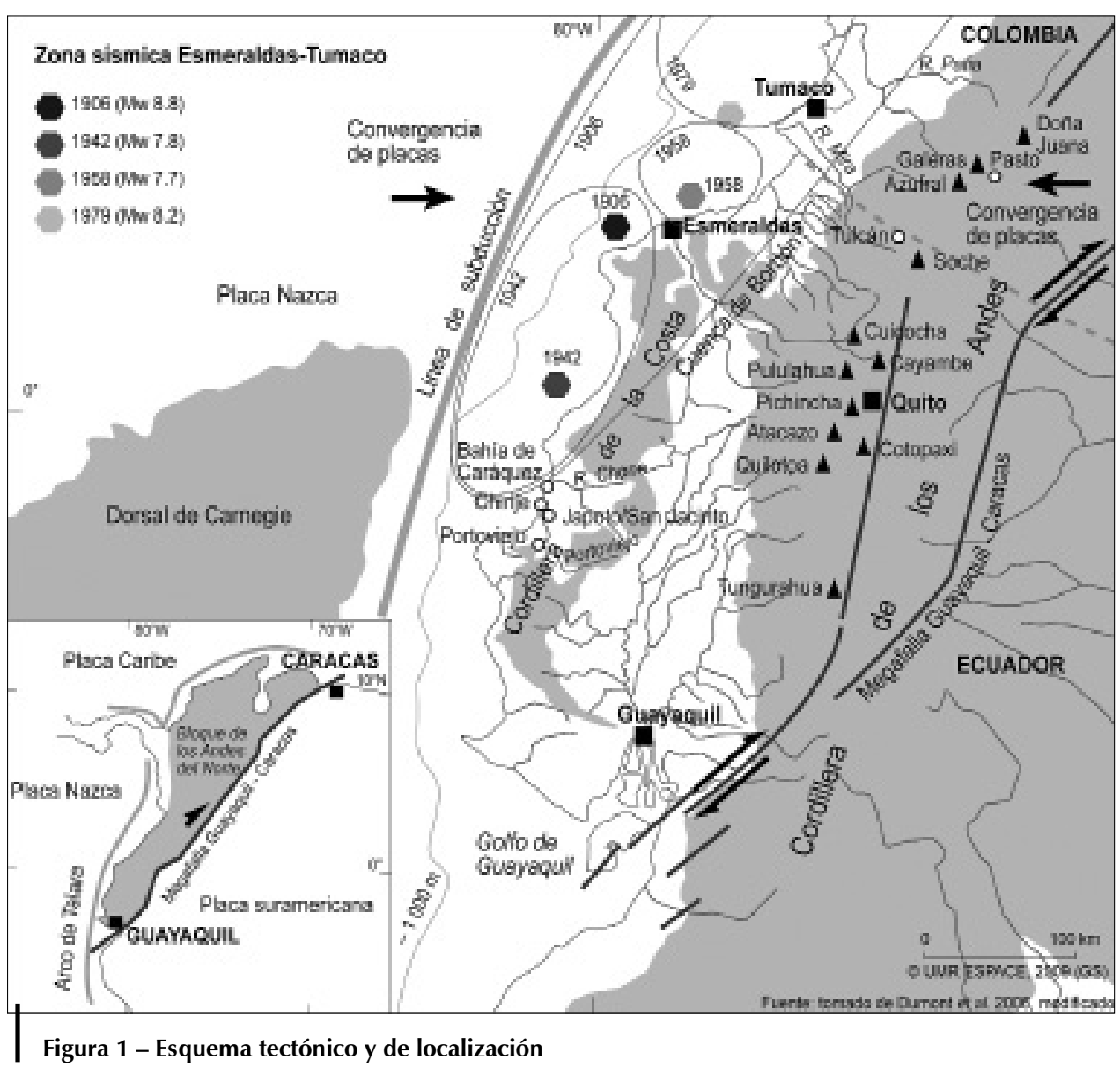

adjuntar a esta dinámica el fenómeno del Niño, ahora bastante bien conocido, cuyas consecuencias han sido, y son todavía, muy importantes para las poblaciones del litoral.

En definitiva, estas grandes amenazas inducen riesgos de segundo orden, tal como deslizamientos y sedimentaciones, inundaciones o sequías, desarrollo de lagunas y de cordones arenosos o su desaparición, modificación de los lechos fluviales, todas amenazas seguramente muy temidas por las poblaciones y grandes obstáculos a su instalación permanente en estas tierras.

\section{UNA TECTÓNICA MUY ACTIVA}

El contacto entre la placa oceánica Nazca al oeste, y la placa americana al este, origina numerosos sismos cuyos registros escritos existen solamente desde la llegada de los españoles. Si se desconocen evidentemente las fechas de los 
sismos anteriores, es evidente que siempre se han producido y han afectado este litoral bien antes del Holoceno. Las medidas de algunos movimientos de fallas actuales (White et al., 2003; Lavenu, 2006) muestran desplazamientos de algunos centímetros al año. Recientemente, Dumont et al. (2006) y Santana et al. (20052006) han puesto en evidencia un movimiento tectónico fechado hacia 32002800 BP (1200-800 AD)1, cuya consecuencia ha sido una modificación drástica del curso del sistema hidrológico Santiago-Cayapas en el norte ecuatoriano (fig. 2), en un momento en el cual pobladores vivían en esta llanura litoral. Menos espectacular, pero tal vez más peligrosa para los habitantes del lugar, se conoce la existencia de tsunamis ligados a los sismos. En Colombia (Martinez, 1992), el sismo de Tumaco en 1979 (fig. 1) ha sido inmediatamente seguido por un tsunami caracterizado por una subida de las aguas de algunos metros, cuya acción destructora hizo muchas víctimas y arrasó con unos pueblos establecidos directamente en la línea de costa. En ambientes sedimentarios finos y arenosos, los sismos producen también movimientos cosísmicos y licuefacción de suelos traduciéndose en hundimientos locales o subsidencias de tamaños más grandes (Martínez, 1992). Es el caso también del sismo de Tumaco en 1979 para el cual se evalúa un hundimiento de unos 1,50 m en el delta del río Mira. Estos fenómenos presentan más consecuencias aún, como es el caso en llanuras aluviales costaneras donde las incisiones de los cauces fluviales son poco marcadas, favoreciendo los bruscos cambios de curso (avulsiones) que pueden ser peligrosos para poblaciones establecidas en las márgenes. Lo mismo pasa, por supuesto, con la penetración brusca de las aguas y la sumersión por el mar de sitios costeros.

\section{EL VOLCANISMO}

Su omnipresencia está directamente ligada a la subducción de la placa pacífica debajo de la placa americana. Los Andes del sur colombiano, como los Andes ecuatorianos cuentan con numerosos volcanes, la mayor parte activos actualmente, y otros que lo han sido durante el Holoceno y antes. Pero, al contrario de las manifestaciones sísmicas, numerosas dataciones han podido ser realizadas sobre las cenizas expulsadas, muy abundantes en el caso dominante de volcanes explosivos, o restos orgánicos contemporáneos de las erupciones. Estas dataciones cubren buena parte del Holoceno2. Los restos de varias lluvias de cenizas se encuentran a lo largo del litoral pacífico, visibles en los cortes naturales y, por supuesto, en las excavaciones arqueológicas (fig. 3). Bien conocido, el predominio de vientos orientados hacia el oeste permite entender la acumulación de importantes capas de cenizas que han podido llegar hasta destruir los cultivos y las habitaciones de los grupos humanos establecidos entonces en el litoral, sobre todo cuando se trata de cenizas riolíticas y dacíticas

1 Hemos utilizado BP por antes del Presente (1950), BC y a. C. por antes del Cristo, A. D. por año Dei

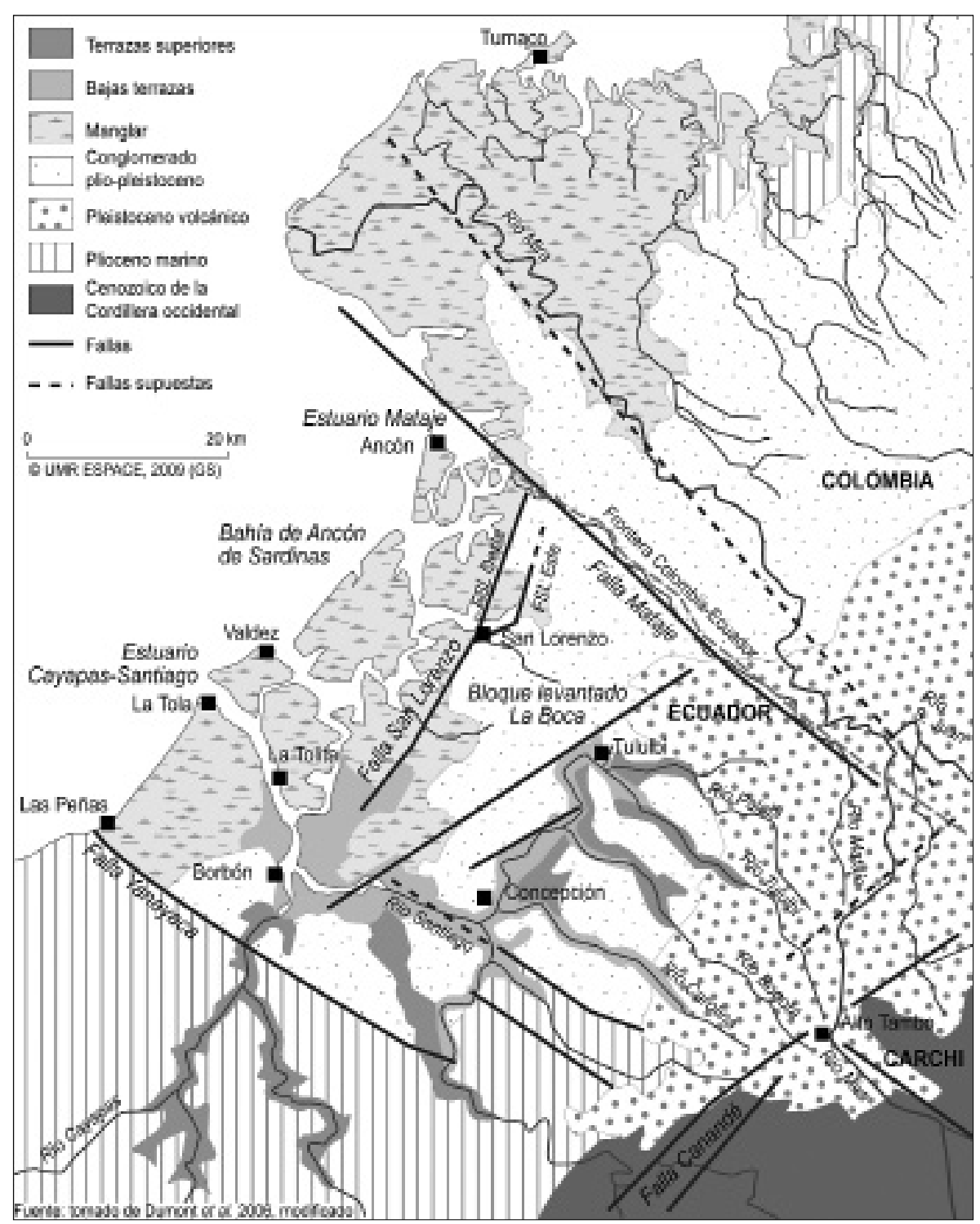

Figura 2 - Accidente Santiago-Cayapas, cambio de curso del río (3200-2800 BP

muy ácidas y desfavorables a los cultivos, caso que se da especialmente, en el Ecuador, con las cenizas provenientes de los volcanes Cotopaxi, Cuicocha y Quilotoa (Hall \& Mothes, 2008)

En Colombia, al sur de los volcanes del Tolima, del Ruiz y del centro de la Cordillera central, se notan las seis erupciones del volcán Galeras entre 4500 BP y el periodo histórico. Se notan también las erupciones de la Doña Juana (2550 


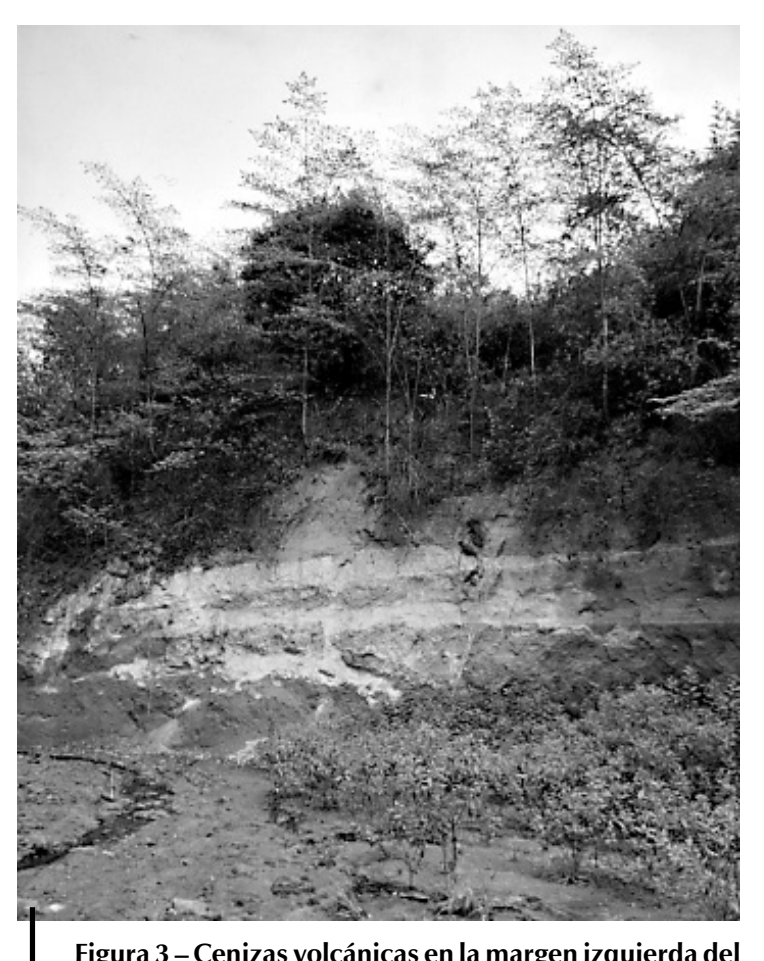

BC) y del Azufral (2000 BC, 1500 BC, 930 BC). Como en el Ecuador, se trata de un volcanismo explosivo emitiendo intensas lluvias de cenizas hasta el litoral pacífico, capaces de hacer desaparecer totalmente cultivos y establecimientos humanos.

En el Ecuador (Mothes, 1998), más documentado, se conocen muchas más erupciones durante el Holoceno Reciente. De norte a sur podemos mencionar: la explosión del Soche alrededor de $8600 \mathrm{BP}$, las dos erupciones del Cuicocha de 3100 y 2900 BP y del Pululahua (o Pululagua) en 6750 y 2400 BP. Esta última erupción habría afectado bastante a la costa pacífica, particularmente al sector de San Mateo cerca de Esmeraldas (Isaacson, 1994; Hall \& Mothes, 2008). Del Cayambé se conocen las erupciones de $2000 \mathrm{BC}$, luego de 1040 y 1290 AD. El Pichincha, siempre muy activo, ha conocido fuertes río Chirije Grande, aguas arriba del sitio arqueológico $\begin{aligned} & \text { siempre muy activo, ha conocido fuertes } \\ & \text { erupciones en } 8150,1400 \text { y } 980 \mathrm{BP}\end{aligned}$ El Atacazo-Ninahuilca (Hidalgo et al., 2008; Hall \& Mothes, 2008; Zeidler \& Isaacson, 1994) es conocido por sus explosiones de 5540, 4440 y 2350-2269 BP, con depósitos comprobados de cenizas en toda la llanura litoral a la altura de Bahía de Caráquez. El Cotopaxi conoce un gran número de erupciones, particularmente las de 3880, 3280, 2640, 400, 230 BC, luego 70, 150, 180, 740, 770, 1130 y 1350 AD. Y, por terminar, el Tungurahua se manifiesta en 7750, 1010, 270 BC, luego en 200 y $730 \mathrm{AD}$.

Según Hall \& Mothes (2008), las mayores crisis explosivas en el Ecuador corresponden a los periodos 4050-2900, 2400 y 980-810 BP, con depósitos de gruesas capas de cenizas suficientes para destruir toda vida; los principales focos suministradores de cenizas en esta época serían, de norte a sur: el Soche, el Pululahua, el Pichincha, el Cotopaxi, el Ninahuilca-Atacazo, el Quilotoa y el Tungurahua.

Desde Tumaco en Colombia, todo el litoral ha sido afectado, sin saber todavía hasta qué punto y cuántas veces han sido marcadas o destruidas las poblaciones. Las espesas capas de cenizas removidas en las laderas por la escorrentía superficia y/o depositadas por los ejes de drenaje (ríos, esteros) constituyen las pruebas del fenómeno. Sin embargo, se desconoce en general, en el Ecuador como en Colombia, el origen preciso y la edad de las cenizas, quedando por hacer todo un trabajo de investigación entre arqueología y ciencias de la tierra. Hasta la fecha, y con respecto al sitio arqueológico de Japoto/San Jacinto, las cenizas parecen ser nítidamente anteriores a la construcción de las tolas observadas.

\section{LOS CAMBIOS CLIMÁTICOS, LAS VARIACIONES DEL NIVEL DEL MAR Y EL FENÓMENO DEL NIÑO}

Se ha agrupado en lo que sigue el conjunto de conocimientos, todavía limitado, que se tiene en la catualidad sobre fenómenos más o menos cíclicos, cambios climáticos y variaciones de niveles marinos observados en la región.

Descritos desde el siglo XVI, El Niño y su contrario, la Niña, se conocen ahora bastante bien.

El fenómeno del Niño resulta de la interacción atmósfera/océano (Merle \& Hisard 1990). Sus consecuencias afectan directamente el continente, particularmente el litoral. Con un alza de la temperatura, su principal característica, a lo largo prácticamente de todo el litoral -menos significativamente en Tumaco y en el extremo norte del litoral ecuatoriano (Rossel, 1997) — es un aumento considerable - a menudo más de $70 \%$ - de las precipitaciones con inundaciones. Con la subida del nivel marino de varias decenas de centímetros (50 centímetros de promedio), el fenómeno se traduce por la aparición de aguas anormalmente cálidas, lo que corresponde al episodio cálido de la Oscilación Sur El Niño, más conocida por su traducción inglesa, El Niño Southern Oscillation o ENSO.

La situación contraria, correspondiendo a la situación «normal», es decir más frecuente, la Niña, es el episodio frío del ENSO, caracterizado, a lo largo de la costa, por subidas de aguas oceánicas frías y ricas en plancton, conocidas bajo el nombre de upwelling. Este fenómeno cíclico, pero con periodos de retorno irregulares (Arteaga et al. 2006), ha afectado seguramente al litoral durante todo o buena parte del Holoceno, y permanece muy activo actualmente. La presencia del Niño a lo largo de todo el Holoceno sigue siendo cuestionada (Clement et al., 2000) mientras otros autores distinguen un Holoceno, con cambios climáticos de términos temporales largos, de las manifestaciones climáticas del Niño de corta duración (Stahl, 1991).

Si se ubica en este espacio de duración más larga para examinar los eventuales cambios climáticos, las observaciones son actualmente muy puntuales, poco numerosas, bastante inseguras y entonces muy discutidas. Desde hace varios decenios de años (Sarma, 1974) se conocen condiciones cambiantes durante el Holoceno, esencialmente alternancias climáticas más húmedas o más secas. Una consideración fundamental es que el entorno natural nunca fue estático sino, al contrario, siempre muy dinámico (Van der Hammen et al., 1996) a lo largo del periodo, rápidamente calificado globalmente de calentamiento siguiendo la última glaciación pleistocénica, y caracterizado entonces con una importante subida del nivel de los océanos.

Más precisamente, se observaría una tendencia al calentamiento climático entre 14000 y 6000 BP, pero con fases de enfriamiento más o menos cortas entre 9000 
y 8000 BP y antes de 6000 BP. Sandweiss (1996, y, más completo, 2003) indica para el norte del Perú un periodo más caliente y más húmedo entre 5500 y 6250 AP (3550 y 4300 a. C). El fenómeno ENSO estaría bien comprobado a partir de $7600 \mathrm{BP}$, con una periodicidad del orden de 10 por siglo, mientras que la frecuencia actual es mucho mayor (hasta 30 ó 40 por siglo, Schulmeister et al., 2006). El optimum climaticum estaría alcanzado alrededor de 6000 BP, con una temperatura algo más elevada que la actual $\left(+1^{\circ}\right)$, seguido por rápidos y cortos cambios climáticos (ahora conocidos como Rapid Climate Change): el optimum climático de la Edad Media (siglos X a XIV), como, más recientemente, la pequeña edad glaciar posterior (siglos XVI-XIX), forman parte de estas oscilaciones. Estos cambios, de algunos centenares de años de duración o a veces mucho menos, parecen presentar una frecuencia más grande desde el Holoceno medio. Quedan inexplicados, seguramente ligados a los ciclos de la órbita terrestre (Eckdahl et al., 2008; Mayewski et al., 2004), pero sus consecuencias han sido tal vez muy importantes para las poblaciones. Los episodios fríos se caracterizan también por sequías, ligados con una actividad solar variable y una migración hacia el sur de la Convergencia intertropical, entre 9000-8000, 6000-5000, 4200-3800, 3500-2500, 1200-1000 y 600-150 BP por ejemplo, y por otros autores entre 3500 y 200 BP, 4200 y 3600 BP, 1200 y 1000 BP. Este último episodio sería contemporáneo del colapso maya y, hacia el fin del siglo VI de nuestra era, 30 años de sequía hubieran afectado peligrosamente la civilización Moche del norte peruano (Mayewski et al., 2004; Weiss \& Bradley, 2001). Sin embargo, esta última hipótesis está siendo ahora bastante criticada (Rosas Dintel, 2007). Globalmente, estos autores consideran que estos periodos corresponden con momentos de ruptura para las culturas y, a veces, con su fenecimiento.

Tocando a las variaciones de nivel que afectan los mares del mundo, se conocen pocas cosas en ese litoral, a excepción de la parte sureña del litoral ecuatoriano (Stothert et al., 2003). En general, se sabe que las costas rocosas, con pendientes subverticales, nunca registran consecuencias importantes de las variaciones de nivel del océano. Al contrario, las costas bajas han tenido que conocer importantes variaciones, y particularmente la pérdida de restos humanos sepultados bajo la subida posglaciar del nivel del mar durante buena parte del Holoceno. Si se considera generalmente que el nivel actual de los océanos se alcanza alrededor de 6000 BP (4000 a. C), Rees et al. (2006) estiman que el nivel actual se establece hacia 4000 BP (2050 a. C). Estas variaciones han afectado también las formaciones vegetales litorales, particularmente los manglares de las costas bajas y areno lodosas: Jaramillo \& Bayona (2000) han demostrado para la costa pacífica norte colombiana cómo el establecimiento de manglares puede ser reciente (2600 BP, 650 AD), tal vez ligado a movimientos tectónicos, mostrando una vez más la complejidad de estas vinculaciones entre cambios climáticos, niveles marinos y tectónica. Sin embargo, la presencia de manglares en esta costa pacífica parece estar demostrada desde hace por lo menos 4500 años. Con frecuencia, cuando existe un levantamiento de la costa, las relaciones océano/tectónica se traducen en el litoral con la presencia de antiguos niveles de playa escalonados (terrazas marinas). Sin embargo, en la porción de litoral entre Tumaco al norte y la desembocadura del río Porto Viejo, en los alrededores de Esmeraldas (Punto Galeras), no se observan tales niveles, al contrario bien visibles inmediatamente al sur (península de Manta). Esta situación se debe al efecto dominante de subsidencia y de hundimiento en esta parte de la costa, tal como se ha notado recientemente (Pedoja et al., 2006).

Sin entrar en detalles, la observación de la dinámica de cordones arenosos marinos actuales, de islas-barreras y de canales de mareas en varias partes de la costa (Tumaco, La Tolita, Esmeraldas) permite reconstrucciones tentativas de lo que ha podido ser el litoral en un pasado reciente, como en el caso del sitio ecuatoriano de Japoto ubicado directamente al norte de la desembocadura del río Porto Viejo (Manabí). El hecho de tener dataciones, sea por el material excavado por los arqueólogos, sea con el tratamiento directo del material orgánico natural, es el elemento fundamental de restitución como se ha logrado cerca del sitio de La Tolita (Tihay \& Usselmann, 1995: fig. 4) donde se ha podido seguir la progresión (agradación) de la costa entre unos 6000 y 500 años BP. En Japoto (fig. 5) no tenemos suficientes dataciones para asegurar el esquema propuesto de ocupación de un espacio acuático que, poco a poco, se llena de sedimentos para encontrarse separado del océano (Usselmann, 2006).

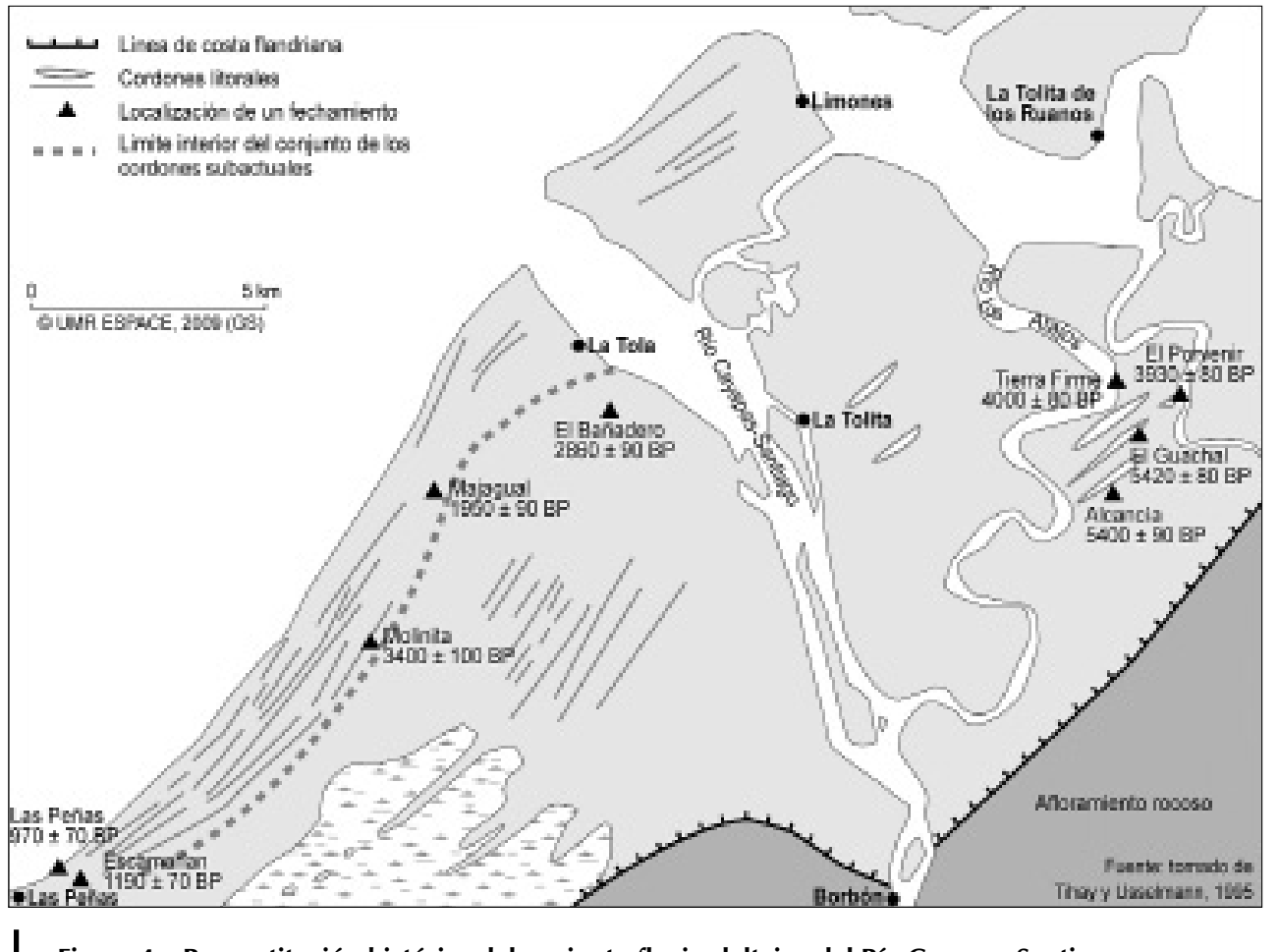

Figura 4 - Reconstitución histórica del conjunto fluvio-deltaico del Río Cayapas-Santiago 


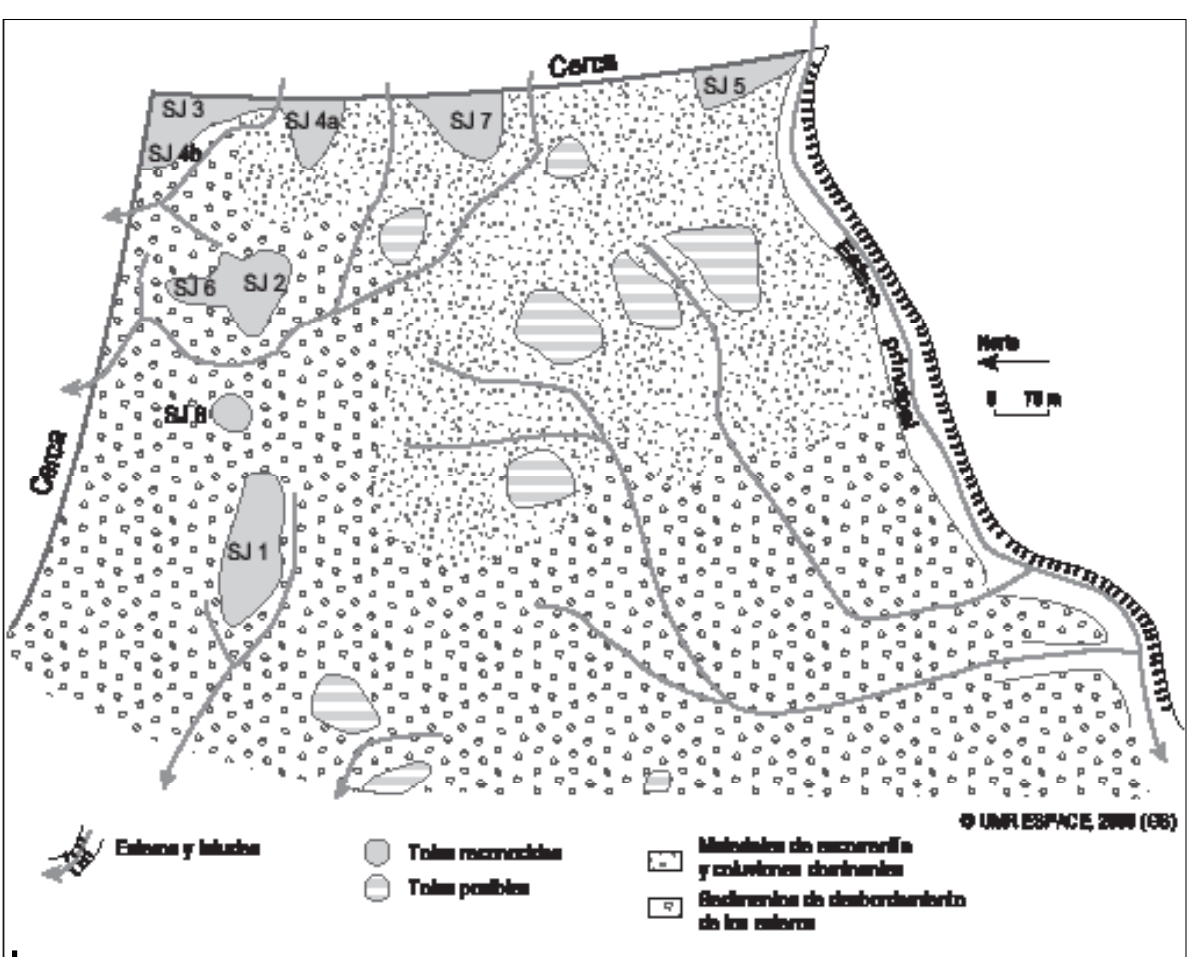

Figura 5 - Esquema del entorno físico de Japoto/San Jacinto

Es evidente sin embargo que la reconstitución de entornos en el caso de sedimentación es mucho más fácil que en el caso de erosión, lo que ocurre cuando se trata de costas rocosas de acantilados como en Chirije (Manabí, Ecuador) donde la línea de costa corta nítidamente las terrazas aluviales donde se encuentra el sitio arqueológico (fig. 6).

Por lo demás, la evolución dinámica de los acantilados en rocas poco resistentes, lutitas en general, se caracteriza por la presencia de múltiples movimientos en masa como en las laderas de las colinas costeñas; estos movimientos se producen tanto por la erosión marina (Usselmann, 2006; Santana et al., 2001; Iriundo, 1992; Plaza et al., 2000) como por las grandes precipitaciones traídas durante los Niños en formaciones ricas en arcillas de gran poder expansivo (tipo montmorillonitas). Estos sedimentos llevados por los cursos de agua, o que llegan directamente al océano, representan una enorme carga, la cual, retomada por la corriente litoral, tiene la capacidad no solamente de construir cordones litorales, sino también de cerrar los estuarios, lo que es el caso del río Chone en Bahía de Caráquez (Tutiven, 1998) y del río Portoviejo.

La erosión caracteriza también algunos sitios de costas bajas: es el caso actualmente de Tumaco (Montagut Cifuentes, 1998), y probablemente el proceso ha existido a lo largo del Holoceno; observaciones similares pueden ser hechas en el litoral de

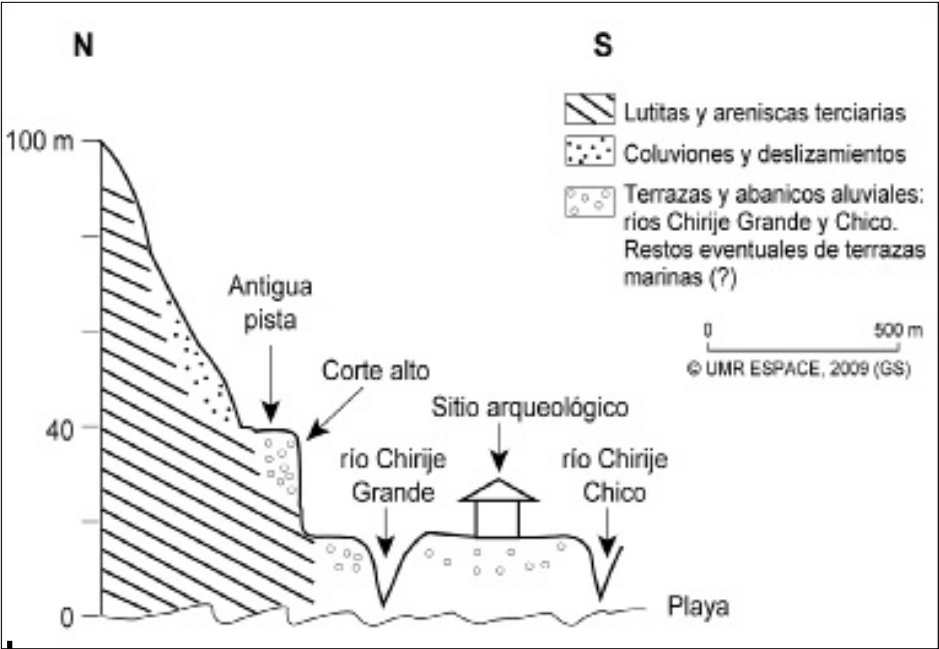

Figura 6 - Sitio Chirije

San Jacinto. Sin embargo, sería un error pensar que han habido solamente erosión o sedimentación en tal o cual parte de la costa: los procesos son más complejos, caracterizados seguramente por la alternancia de periodos de erosión o de sedimentación. Solamente la correlación entre formas o sedimentos y dataciones precisas - aprovechando particularmente las excavaciones arqueológicaspueden permitir una reconstitución precisa de la evolución.

\section{CONCLUSIÓN}

Frente a la complejidad del problema, es evidente que solo una buena y planeada cooperación entre arqueólogos e investigadores del medio ambiente (geomorfólogos, geólogos, pero también botanistas y especialistas de la fauna) puede lograr una reconstitución correcta de lo que ha sido una ocupación antigua (o varias ocupaciones antiguas) de este litoral colombo ecuatoriano, siempre con el uso de dataciones absolutas. Los proyectos arqueológicos actuales, como los de años anteriores, han generalizado esta percepción. Sin embargo, queda mucho por hacer, y son de esperar aportes revolucionarios en los años venideros. 


\section{Referencias citadas}

ARTEAGA, K., TUTASI, P. \& JIMÉNEZ, R., 2006 - Climatic variability related to El Niño in Ecuador, a historical background. Advances in Geosciences, 6: 237-241.

BOUCHARD, J.-F., 2008 - Japoto, une métropole régionale tardive dans la province côtière du Manabi (Équateur). Les Nouvelles de l'Archéologie, 111-112: 89-94.

CLEMENT, A. C., SEAGER, R. \& CANE, M. A., 2000 - Suppression of El Niño during the mid-Holocene by changes in the Earth's orbit. Paleoceanography, 15 (6): 731-737.

DUMONT, J.-F., SANTANA, E., VALDEZ, F., TIHAY, J.-P., USSELMANN, P., ITURRALDE, D. \& NAVARRETE, E., 2006 - Fan beheading and drainage diversión as evidence of a 3200-2800 BP earthquake event in the Esmeraldas-Tumaco seismic zone: A case study for the effects of great subduction earthquakes. Geomorphology, 74: 100-123.

ECKDAHL, E. J., FRITZ, S. C., BAKER, P. A., RICSBY, C. A. \& COLEY, K., 2008 - Holocene multidecadal -to millennial- scale hydrologic variability on the South American Altiplano. The Holocene, 18: 867-876.

HALL, M. \& MOTHES, P., 2008 - Volcanic impediments in the progressive development of pre-Columbian civilizations in the Ecuadorian Andes. Journal of Volcanology and Geothermal Research,176 (3): 344-355.

HIDALGO, S., MONZIER, M., ALMEIDA, E., CHAZOT, G., EISSEN, J.-P., VAN DER PLICHT, J. \& HALL, M. L., 2008 - Late Pleistocene and Holocene activity of the AtacazoNinahuilca Volcanic Complex (Ecuador). Journal of Volcanology and Geothermal Research, 176 (1): 16-26.

IRIONDO, M., 1992 - Dinámica litoral en la costa ecuatoriana durante el Holoceno superior; Lima, ORSTOM/CONCYTEC. Paleo ENSO Records Internacional Symposium. Ext. Abstracts, 155-158.

ISAACSON, J., 1994 - Volcanic sediments in archaeological contexts from western Ecuador In: Regional Archaeology in Northern Manabí, Ecuador (J. Zeidler \& D. Pearsall, eds.): 1, 8, 131-140; Pittsburg: Univ. Pittsburg Memoirs Latin American Archaeology.

JARAMILLO, C. \& BAYONA, G., 2000 - Mangrove distribution during the Holocene in Tribuga Gulf, Colombia. Biotropica, 32 (1): 14-22

LAVENU, A., 2006 - Neotectónica de los Andes entre $1^{\circ} \mathrm{N}$ y $47^{\circ} \mathrm{S}$ (Ecuador, Bolivia y Chile): una revisión. Revista de la Asociación de Geólogos Argentinos, 61 (4): 504-524.

MARTINEZ, M. J. O., 1992 - Geomorfología de la costa del Pacífico colombiano y aspectos relativos a su estabilidad; Lima, ORSTOM/CONCYTEC. Paleo ENSO Records International Symposium. Ext. Abstracts, 193-196.

MAYEWSKI, P. A., ROHLING, E. E., STAGER, J. C., KARLEN, W., MAASCH, K.A., MEEKER, L. D., MEYERSON, E. A., GASSE, F., VAN KREVELD, S., HOLMGREN, K., LEE-THORP, J., ROSQVIST, G., PACK, F., STAUBWASSER, M., SCHNEIDER, R. R. \& STEIG, E. J., 2004 - Holocene climate variability. Quaternary Research, 62: 243-255.

MERLE, J. \& HISARD, P., 1990 - Interactions océan-atmosphère dans les Tropiques. Annales de Géographie, 553: 273-290.

MONTAGUT CIFUENTES, E. A., 1998 - Principales cambios en la línea de costa del municipio de San Andrés de Tumaco. Boletín Científico, 7: 67-77; Tumaco: Centro Control Contaminación del Pacífico.

MOTHES, P. (ed.), 1998 - Actividad volcánica y pueblos precolombinos en el Ecuador, 205 pp.; Quito: Abya Yala.
PEDOJA, K., ORTLIEB, L., DUMONT, J.-F., LAMOTHE, M., GHALEB, B., AUCLAIR M. \& LABROUSSE, B., 2006 - Quaternary coastal uplift along the Talara Arc (Ecuador, Northern Peru) from new marine terrace data. Marine Geology, 228 (1-4): 73-91.

PLAZA, G., GALÁRRAGA, R., VALVERDE, J., PROANO, O. \& JIMÉNEZ, E., $2000-$ Zonificación del peligro por deslizamientos, flujos de lodos y escombros y sismos. El caso de la ciudad de Bahía de Caráquez, Ecuador; Caracas: Facultad de Ingeniería

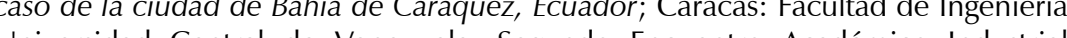
Universidad Central de Venezuela. Segundo Encuentro Académico Industrial,
Jornadas de investigación, Resumen, 610-611.

EES, S. A., OPDYKE, B. N., WILSON, P. A., FIFIELD, L. K. \& LEVCHENKO, V., $2006-$ Holocene evolution of the granite based Lizard Island and MacGilliway Coral Reef systems. Northern Great Barrier Reef, Coral Reefs, 25 (4): 555-565.

ROSAS DINTEL, M., 2007 - Nuevas perspectivas acerca del colapso Moche en el Bajo equetepeque. Resultados preliminares de la segunda campaña de investigación del proyecto arqueológico Cerro Chepen. Bulletin de l'Institut Français d'Etudes Andines, 36 (2): 221-240.

ROSSEL, F., 1997 - Influence du Niño sur les régimes pluviométriques de l'Équateur, 289 pp. + anexos; Montpellier: Université Montpellier II, Tesis de Doctorado.

SANDWEISS, D. H., 1996 - Mid-Holocene cultural interaction between the North coast of Peru and Ecuador. Latin American Antiquity, 7 (1): 41-50.

SANDWEISS, D. H., 2003 - Terminal Pleistocene through Mid-Holocene archaeological sites as paleoclimatic archives for the Peruvian coast. Palaeogeography, Palaeoclimatology, Palaeoecology, 194: 23-40.

SANTANA, E., DUMONT, J.-F. \& KING, A., 2001 - Los efectos del fenómeno El Niño en la ocurrencia de una alta taza de erosión costera en el sector de Punta Gorda, Esmeraldas. Acta Oceanografica del Pacífico, 11 (1): 1-8.

SANTANA, E., DUMONT, J.-F., VALDEZ, F., PAZMIÑO, N., TIHAY, J.-P., USSELMANN, P. \& LOPEZ, E., 2005-2006 - Método morfo-estructural para la identificación de paleo eventos tecto-sísmicos: aplicación a la zona costera de San Lorenzo, Norte de Ecuador. Acta Oceanografica del Pacífico, 13 (1): 227-241.

SARMA, A. V. N., 1974 - Holocene Paleoecology of South Coastal Ecuador. Proceedings of the American Philosophical Society, 118 (1): 93-134.

SCHULMEISTER, J., RODBELL, D. T., GAGEN, M. K. \& SELTZER, G. O., 2006 Interhemispheric Linkages in climate change: paleoperspectives for future climate change. Climate of the Past, 2: 167-185.

STAHL, P. W., 1991 - Arid Landscapes and environmental Transformations in ancient southwestern Ecuador. World Archaeology, 22 (3): 346-359.

STOTHERT, K. E., PIPERNO, D. R. \& ANDRES, T. C., 2003 - Terminal Pleistocene/Early Holocene human adaptation in coastal Ecuador: the Las Vegas evidence. Quaternary International, 109-110: 23-43. South America: Long and winding roads for the first Americans at the Pleistocene/Holocene transition.

TIHAY, J.-P. \& USSELMANN, P., 1995 - Medio ambiente y ocupación humana en el litoral pacífico colombo-ecuatoriano (M. Guinea, J.-F. Bouchard \& J. Marcos, eds.): 377 400; Quito: Bibliot. Abya Yala 21.

TUTIVEN UBILLA, I., 1998 - Variaciones morfológicas y batimétricas de la línea de costa en el estuario del río Chone, producidas por los eventos ENSO. Bulletin de l'Institut Français d'Études Andines, 27 (3):557-563.

USSELMANN, P., 2006 - Dinámica geomorfológica y medio ambiente en los sitios arqueológicos Chirije y San Jacinto/Japoto (costa del Manabí central, Ecuador). Bulletin de l'Institut Français d'Études Andines, 35 (3): 257-264. 
VAN DER HAMMEN, T. \& HOOGHIEMSTRA, H., 1996 - The Abra stadial: A Younger Dryas equivalent in Colombia. Quaternary science Reviews, 14: 841- 851.

WEISS, H. \& BRADLEY, R. S., 2001 - What Drives Societal Collapse? Science, 291: 609610.

WHITE, S. M., TRENKAMP, R. \& KELLOGG, J. N., 2003 - Recent crustal deformation and the earthquake cycle along the Ecuador-Colombia subduction zone. Earth and Planetary Science Letters, 216: 231-242.

ZEIDLER, J. A. \& PEARSALL, D. M., 1994 - Regional Archaeology in Northern Manabi, Ecuador, 224 pp.; Pittsburg/Quito: Univ. of Pittsburgh. Memoirs in Latin American Archaeology, 1. 